\title{
Effects of sevoflurane on tight junction protein expression and PKC- $\alpha$ translocation after pulmonary ischemia-reperfusion injury
}

\author{
Jun Chai ${ }^{1}$, Bo Long ${ }^{1}$, Xiaomei $\mathrm{Liu}^{2}$, Yan $\mathrm{Li}^{1}$, Ning $\mathrm{Han}^{1}$, Ping Zhao ${ }^{1}$ and Weimin Chen ${ }^{1}$
}

Pulmonary dysfunction caused by ischemia-reperfusion injury is the leading cause of mortality in lung transplantation. We aimed to investigate the effects of sevoflurane pretreatment on lung permeability, tight junction protein occludin and zona occludens 1 (ZO-1) expression, and translocation of protein kinase C (PKC)- $\alpha$ after ischemia-reperfusion. A lung ischemia-reperfusion injury model was established in $\mathbf{9 6}$ male Wistar rats following the modified Eppinger method. The rats were divided into four groups with 24 rats in each group: a control (group C), an ischemia-reperfusion group (IR group), a sevoflurane control group (sev-C group), and a sevoflurane ischemia-reperfusion group (sev-IR group). There were three time points in each group: ischemic occlusion for $45 \mathrm{~min}$, reperfusion for $60 \mathrm{~min}$ and reperfusion for $120 \mathrm{~min}$; and there were six rats per time point. For the 120-min reperfusion group, six extra rats underwent bronchoalveolar lavage. Mean arterial pressure (MAP) and pulse oxygen saturation $\left(\mathrm{SpO}_{2}\right)$ were recorded at each time point. The wet/dry weight ratio and lung permeability index (LPI) were measured. Quantitative RT-PCR and Western blot were used to measure pulmonary occludin and ZO-1, and Western blot was used to measure cytosolic and membranous PKC- $\alpha$ in the lung. Lung permeability was significantly increased after ischemia-reperfusion. Sevoflurane pretreatment promoted pulmonary expression of occludin and ZO-1 after reperfusion and inhibited the translocation of PKC- $\alpha$. In conclusion, sevoflurane pretreatment alleviated lung permeability by upregulating occludin and ZO-1 after ischemia-reperfusion. Sevoflurane pretreatment inhibited the translocation and activation of PKC- $\alpha$, which also contributed to the lung-protective effect of sevoflurane.

Experimental \& Molecular Medicine (2015) 47, e167; doi:10.1038/emm.2015.27; published online 5 June 2015

\section{INTRODUCTION}

Pulmonary dysfunction caused by ischemia-reperfusion injury is the leading cause of mortality in lung transplantation, and it is also one of the major complications of perioperative events such as shock, trauma and cardiopulmonary bypass. The lung injury after ischemia-reperfusion mainly manifests as increased permeability of lung tissue and increased lung water and protein content. Alveolar-capillary barrier dysfunction is the main cause of lung ischemia-reperfusion injury. The integrity of lung capillary endothelial cells and alveolar epithelial cells is critical to maintain the function of the respiratory barrier, ${ }^{1}$ which is a semipermeable membrane between capillary, pulmonary interstitial tissue and alveolar space. Alveolarcapillary barrier dysfunction allows a variety of liquids and macromolecules to penetrate into the lung interstitial tissue and alveoli.
Sevoflurane is an inhaled anesthetic commonly used clinically. It has a low blood/gas partition coefficient and the advantages of anesthesia induction, quick recovery and hemodynamic stability. Sevoflurane has anti-inflammatory properties, and pretreatment with sevoflurane can protect lung tissue from ischemia-reperfusion injury, ${ }^{2,3}$ but the mechanism of its protective effect on reperfusion injury is not fully understood.

Tight junctions are a type of cellular connection located at the top of pulmonary capillary endothelial cells and epithelial cells. They are gateways that prevent solute diffusion through intercellular spaces, and their integrity is important to maintain pulmonary vascular permeability. ${ }^{4}$ Tight junctions are composed of a variety of transmembrane proteins and several peripheral membrane proteins that are closely linked to the cytoskeleton. ${ }^{5}$ Among them, the most important are occludin and zona occludens 1 (ZO-1). ZO-1 is the bridge connecting

\footnotetext{
${ }^{1}$ Department of Anesthesiology, Shengjing Hospital of China Medical University, Shenyang City, China and ${ }^{2}$ Central Laboratory of Shengjing Hospital, China Medical University, Shenyang City, China

Correspondence: Professor P Zhao, Department of Anesthesiology, Shengjing Hospital of China Medical University, No. 36, Sanhao Street, Heping District, Shenyang City 110004, China.

E-mail: zhaop@sj-hospital.org
}

Received 23 September 2014; revised 7 January 2015; accepted 9 January 2015 
occludin and the cytoskeleton. Changes in occludin and ZO-1 expression are closely related to pulmonary tissue permeability.

Protein kinase $\mathrm{C}(\mathrm{PKC})$ activation is the main cause leading to increased microvascular permeability and lung injury. ${ }^{6,7}$ The subtype of PKC- $\alpha$ is mainly expressed in vascular endothelial cells. Increased vascular permeability in ischemia-reperfusion or inflammation is closely related to PKC- $\alpha$ activation. ${ }^{8,9}$ Under no stimulation, PKC- $\alpha$ is inactive in the cytoplasm and its content is very low. Under stimulation, PKC- $\alpha$ translocates from the cytoplasm to the cell membrane and cytoskeleton. This shift is an important sign of PKC- $\alpha$ activation. It is not yet known whether the PKC- $\alpha$ shift and activation are involved in the lung tissue permeability decline after sevoflurane pretreatment in a lung ischemiareperfusion model.

The purpose of this study is to investigate whether sevoflurane pretreatment can reduce pulmonary tissue permeability after ischemia-reperfusion and to determine the mechanism by which sevoflurane affects tight junctions and PKC- $\alpha$.

\section{MATERIALS AND METHODS}

\section{Animal models}

Ninety-six male Wistar rats, weighing 250-350 g (China Medical University Experimental Animal Center), were placed in a relatively clean environment $\left(22^{\circ} \mathrm{C}, 55 \%\right.$ humidity, circadian rhythm), housed separately, and fed a standard diet with water ad libitum. All animal experiments met the requirements of the Federation of European Laboratory Animal Associations.

A lung ischemia-reperfusion injury model was established based on the modified Eppinger method. ${ }^{10}$ After anesthesia, the animals were placed at supine position, the right femoral vein was separated, and a continuous infusion of $0.9 \%$ sodium chloride was set up. A middle-neck incision was made, and the trachea and left carotid artery were freed from surrounding tissues. Tracheotomy, endotracheal intubation and carotid artery intubation were performed, and then the animals were connected to ventilator (Teli Anesthesia and Respiration Equipment Co., Jiangxi, China) for mechanical ventilation $\left(\mathrm{FiO}_{2}=100 \%, \mathrm{Vt}=10-15 \mathrm{ml} \mathrm{kg}^{-1}, f=70-90\right.$ times per min). Using a gas analyzer (Datex-Ohmeda Co., Helsinki, Finland), breathing parameters were adjusted to maintain $\mathrm{PaCO}_{2}$ at $35-45 \mathrm{~mm} \mathrm{Hg}$ $(1 \mathrm{kP}=7.5 \mathrm{~mm} \mathrm{Hg})$. The left carotid artery was connected to an arterial transducer to continuously monitor arterial blood pressure and collect blood samples, and vecuronium was continuously infused at $0.6 \mathrm{mg} \mathrm{kg}^{-1} \mathrm{~h}^{-1}$ to maintain anesthesia. In the sevoflurane groups, sevoflurane (batch number: 44744950, Baxter, Berkshire, UK) was inhaled at 1 minimum alveolar concentration $(2.2 \%)$ as adjusted by a gas monitor. Thirty minutes after mechanical ventilation, the rats were put in the supine position, the chest was opened through the left fifth intercostal space, the left pulmonary ligament was transected, and the left hilum was freed. Five minutes after intravenously injecting $50 \mathrm{U}$ heparin, the left hilum (including the left bronchus and the left pulmonary artery and vein) was occluded with a noninvasive vascular clip for $45 \mathrm{~min}$ and then released to form reperfusion. The animals were euthanized at the different time points and specimens were collected.

\section{Experimental groups and treatment}

The Wistar rats were randomly divided into four groups with 24 animals in each group. In the control group (group C), the chest was opened, and the left hilum was freed but not occluded. In the ischemia-reperfusion group (group IR), the left hilum was occluded for $45 \mathrm{~min}$ and then released to form perfusion. In the sevoflurane group (group sev), the animals were put on sevoflurane for $30 \mathrm{~min}$, and the left hilum was freed but not occluded. In the sevoflurane/ ischemia-reperfusion group (group sev-IR), the animals were put on sevoflurane for $30 \mathrm{~min}$, and then the left hilum was occluded for $45 \mathrm{~min}$ and then released to form perfusion. In each group, there were three time points: ischemic occlusion for $45 \mathrm{~min}$, reperfusion for $60 \mathrm{~min}$ and reperfusion for $120 \mathrm{~min}$. At each time point there were six rats. There were six extra rats at the $120 \mathrm{~min}$ time point for collecting bronchoalveolar lavage fluid (BALF). At each time point, MAP was recorded and blood samples were collected for measuring $\mathrm{PaO}_{2}$.

\section{Detection of pulmonary vascular permeability}

After its wet weight $(W)$ was recorded, $100 \mathrm{mg}$ of lung tissue was dried in an oven at $60^{\circ} \mathrm{C}$ for $48 \mathrm{~h}$ to reach a constant weight, which is called the dry weight $(D)$. The ratio of $W / D(W / D=W / D \times 100 \%)$ was calculated. The Bradford method was used to measure the total serum protein content and BALF protein content. Lung permeability index was calculated (lung permeability index $(\%)=$ BALF protein/serum protein $\times 100)$.

\section{Detection of occludin and ZO-1}

One hundred milligrams of frozen lung tissue was homogenized in buffer A $(0.32 \mathrm{M}$ sucrose, $5 \mathrm{~mm}$ Tris- $\mathrm{HCl} \mathrm{pH} \mathrm{7.5,} 120 \mathrm{~mm} \mathrm{KCl,} 1 \mathrm{~mm}$ EDTA, $0.2 \mathrm{~mm}$ phenylmethylsulfonylfluoride, $1 \mu \mathrm{g} \mathrm{ml}^{-1}$ leupeptin, 1 $\mu \mathrm{g} \mathrm{ml}^{-1}$ pepstatin and $1 \mu \mathrm{g} \mathrm{ml}^{-1}$ aprotinin) and spun at $4{ }^{\circ} \mathrm{C}$, $12890 \mathrm{~g}$ for $10 \mathrm{~min}$. The supernatant was collected, consisting of the cytoplasmic protein. The precipitate was incubated in buffer B $(20 \mathrm{~mm}$ HEPES ( $\mathrm{pH} 7.5$ ), 10\% glycerol, 2\% Triton X-100, 1 mM EDTA, $0.2 \mathrm{~mm}$ PMSF, $1 \mu \mathrm{g} \mathrm{ml}^{-1}$ leupeptin, $1 \mu \mathrm{g} \mathrm{ml}^{-1}$ pepstatin and $1 \mu \mathrm{g} \mathrm{ml}^{-1}$ aprotinin) at $4{ }^{\circ} \mathrm{C}$ overnight and spun at $12890 \mathrm{~g}$ for $10 \mathrm{~min}$. The supernatant was collected, consisting of the membrane proteins. The protein concentrations were measured by the Bradford method. Western blotting was performed to detect occludin and ZO-1. The primary antibodies (goat anti-mouse ZO-1 polyclonal antibody and goat anti-mouse occludin polyclonal antibody, both 1:200) were purchased from Santa Cruz Biotechnology (Santa Cruz, CA, USA). The secondary antibody (horse anti-goat horseradish peroxidase-conjugated polyclonal antibody, 1:2000) was purchased from Zhongshan Biotechnology Inc. (Beijing, China). The data were analyzed with a gel image analysis system, and the relative level of protein expression was calculated as the percentage of optical density in each experimental group to the optical density in group C.

\section{Determination of occludin and ZO-1 mRNA expression}

Total RNA was extracted using TRIzol reagent from $100 \mathrm{mg}$ lung tissue, and the total RNA concentration was measured using a NanoVue (GE Healthcare, Piscataway, NJ, USA). One microgram of total RNA was used for reverse transcription. Subsequently, fluorescent quantitative PCR was implemented with the parameters $94^{\circ} \mathrm{C}$ for $30 \mathrm{~s}$, 35 cycles of $55^{\circ} \mathrm{C}$ for $30 \mathrm{~s}$ and $72{ }^{\circ} \mathrm{C}$ for $45 \mathrm{~s}$, and elongation at $72^{\circ} \mathrm{C}$ for $7 \mathrm{~min}$. A gel image analysis system was used to measure the relative levels of occludin and ZO-1 using $\beta$-actin as the internal control.

\section{Immunohistochemical detection of occludin and ZO-1}

The samples were deparaffinized in xylene and dehydrated in a gradient of ethanol dilutions. A proteolytic enzyme solution was used for antigen retrieval. The occludin and ZO-1 antibodies were the same 
as above. The semiquantitative analysis of occludin and ZO-1 was performed using a computer image analysis system.

\section{PKC- $\alpha$ expression in the cell membrane and cytoplasm}

The preparation of cytoplasmic and membrane proteins was the same as above. The protein concentration was measured using the Bradford method. Western blotting was performed to detect occludin and ZO-1. Rabbit anti-mouse PKC- $\alpha$ antibody was purchased from Santa Cruz Biotechnology (Santa Cruz, CA, USA) (1:200) and the secondary goat-anti-rabbit antibody was purchased from Zhongshan Biotechnology Inc (Beijing, China) (1:2000). The data were analyzed with a gel image analysis system. The relative level of PKC- $\alpha$ protein was calculated as the percentage of optical density in each experimental group to optical density in group C.

\section{Statistical analysis}

All data were analyzed using SPSS 11.5 software (SPSS Inc., Chicago, IL, USA) and are presented as the mean \pm standard deviation $(x \pm s)$. The comparison between groups was analyzed using analysis of variance, and paired comparisons were analyzed using the StudentNewman-Keuls test. $P<0.05$ was considered statistically significant.

\section{RESULTS}

\section{Sevoflurane pretreatment reduces lung permeability}

Compared with those in group $\mathrm{C}$ and group sev, the W/D ratio and lung permeability index at reperfusion 60 and $120 \mathrm{~min}$ significantly increased in group IR and group sev-IR $(P<0.05)$, indicating that reperfusion led to increased wet weight and lung permeability. At the same time points, the W/D ratio and lung permeability index were significantly lower in the sev-IR group than in the IR group $(P<0.05)$, indicating that sevoflurane may have a protective effect on ischemia-reperfusion injury (Figure 1).

\section{Sevoflurane pretreatment increases occludin and ZO-1} mRNA and protein expression after ischemia-reperfusion Both RT-PCR and western blotting were used to examine the effect of sevoflurane pretreatment on occludin and ZO-1 expression. As shown in Figure 2, compared with group C and group sev-C, occludin and ZO-1 mRNAs were significantly decreased in group IR and group sev-IR $(P<0.05)$, and occludin and ZO-1 mRNAs at the 120-min reperfusion were significantly lower than those at the 60-min reperfusion $(P<0.05)$, indicating that sevoflurane pretreatment significantly increased occludin and ZO-1 mRNA expression. Similarly, in the IR group, occludin and ZO-1 protein were significantly decreased after reperfusion $(P<0.05)$. At $120 \mathrm{~min}$, occludin and ZO-1 protein were lower than those after reperfusion for $60 \mathrm{~min}(P<0.05)$, indicating that long-term reperfusion may decrease occludin and ZO-1 protein expression (Figure 3). Compared with the IR group, occludin and ZO-1 were increased in the sev-IR group at both 60 and $120 \mathrm{~min}(P<0.05$; Figure 3$)$, indicating that sevoflurane may have a protective effect on ischemia-reperfusion injury.

Immunohistochemistry was used to confirm the localization of occludin and ZO-1, and further quantify their expression. As shown in Figure 4, occludin and ZO-1 were mainly detected in cytoplasmic membranes and peripheral cytoplasm in alveolar epithelial and endothelial cells, and the staining was stronger at the junction of adjacent cells. In the IR group, occludin and ZO-1 were significantly decreased $(P<0.05)$, and at the 120-min reperfusion, occludin and ZO-1 expression were significantly lower than at $60 \mathrm{~min}(P<0.05)$. Sevoflurane pretreatment significantly increased occludin and $\mathrm{ZO}-1$ protein in lung tissue after reperfusion $(P<0.05)$. The average optical density is shown in Figures $4 c$ and d.

\section{Sevoflurane inhibits PKC- $\alpha$ translocation after reperfusion} In both group IR and group sev-IR, cytoplasmic PKC- $\alpha$ expression decreased with the progress of reperfusion $(P<0.05)$. At the same time, membrane $\mathrm{PKC}-\alpha$ protein expression significantly increased $(P<0.05)$, as shown in Figure $5 \mathrm{a}$. With sevoflurane pretreatment, cytoplasm PKC- $\alpha$ significantly increased $(P<0.05)$ and membrane PKC- $\alpha$ protein expression significantly decreased (as shown in Figure $5 b$ ).

\section{DISCUSSION}

In this study, we investigated the effect of sevoflurane on lung tissue permeability in ischemia-reperfusion injury. The lung
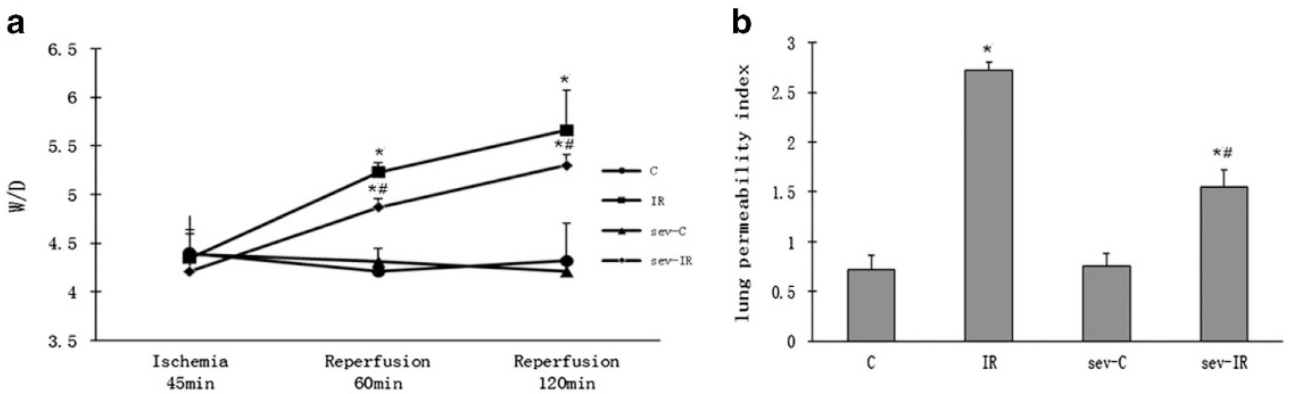

Figure 1 Effect of sevoflurane pretreatment on the vascular permeability of lung tissues after ischemia/reperfusion (I/R). The control group (group C) underwent sham operation. The ischemia-reperfusion group (group IR) underwent left hilum occlusion for 45 min followed by perfusion. The sevoflurane group (group sev-C) underwent sham operation and received sevoflurane for 30 min. The sevoflurane/ischemiareperfusion group (group sev-IR) underwent the same procedure as group IR but was pretreated with sevoflurane for 30 min. The wet-to-dry (W/D) ratio (a) and the lung permeability index (b) were determined as detailed in the Methods section. The data shown are the mean \pm s.e.m. of at least three independent experiments ( $n=6$ in each group). ${ }^{*} P<0.05$ vs group $C$ and group sev-C; ${ }^{\#} P<0.05$ vs group IR. 

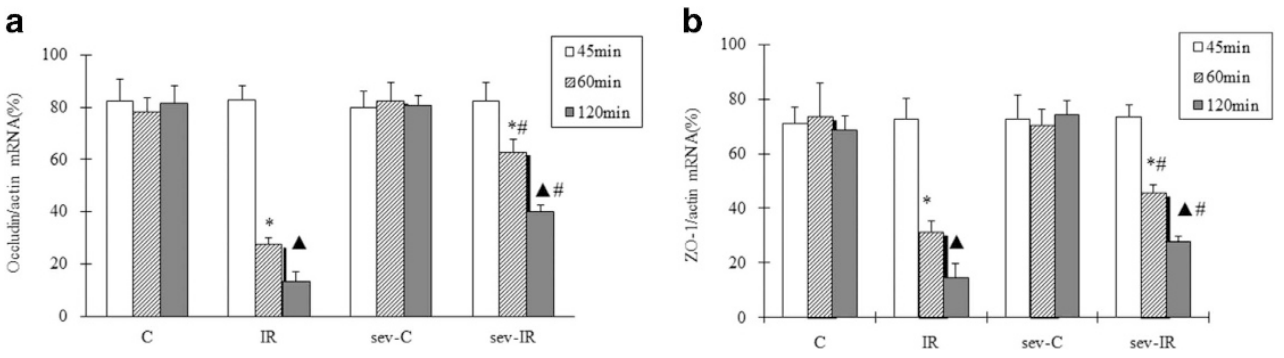

Figure 2 Sevoflurane pretreatment increases occludin and ZO-1 mRNA levels after ischemia/reperfusion. The animals were treated as in Figure 1, and the mRNA transcript levels of occludin (a) and ZO-1 (b) were determined by RT-PCR. The data are presented as the mean \pm s.d. of at least three independent experiments ( $n=6$ in each group). ${ }^{*} P<0.05$ vs group $C$ and group sev- $C$ at reperfusion 60 min; $\boldsymbol{\Delta} P<0.05$ vs group $C$ and group sev-C at 120 -min reperfusion; ${ }^{\#} P<0.05$ vs group IR at 60 - and 120 -min reperfusion.

a

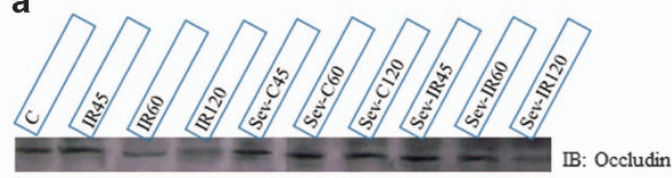

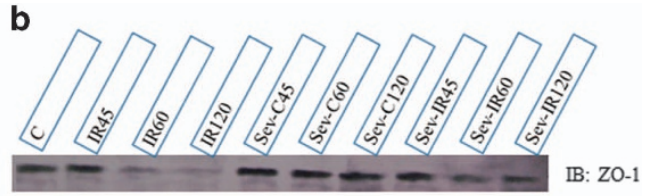
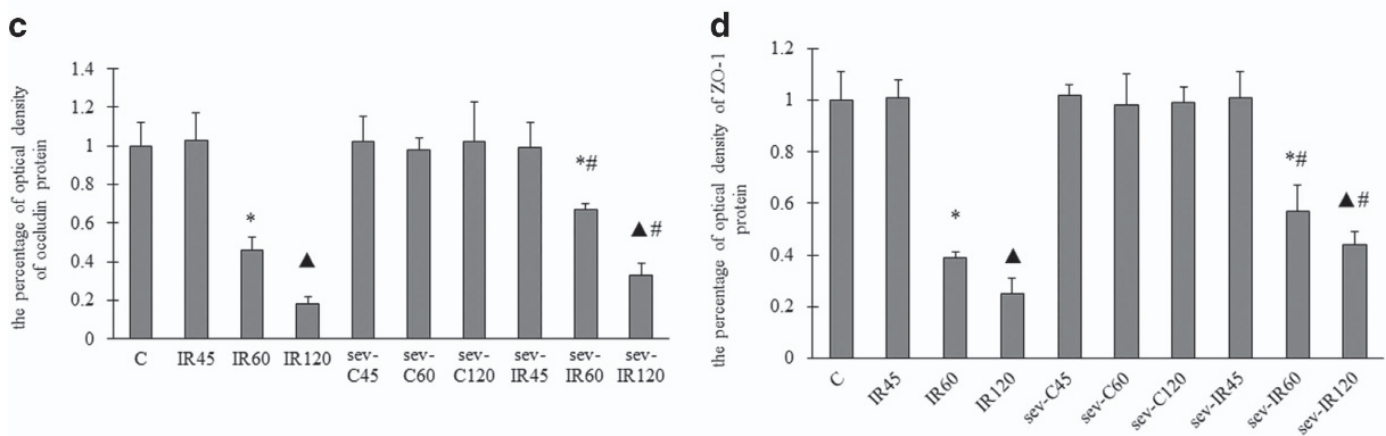

Figure 3 Sevoflurane pretreatment increases occludin and ZO-1 protein expression after ischemia-reperfusion. The animals were treated as in Figure 1, and the protein levels of occludin (a) and ZO-1 (b) were determined by Western blot. Occludin and ZO-1 expression was normalized against $\beta$-actin and is expressed as fold increase compared with group C (set as 1) (c) and (d). Representative Western blots are shown in $\mathbf{a}$ and $\mathbf{b}$. The data are presented as the mean \pm s.d. of at least three independent experiments ( $n=6$ in each group); ${ }^{*} P<0.05$ vs group $C$ and group sev-C at 60 -min reperfusion; $\boldsymbol{\Delta} P<0.05$ vs group $C$ and group sev-C at 120 -min reperfusion; ${ }^{\#} P<0.05$ vs group IR at 60 and 120-min reperfusion.

permeability index and W/D ratio are indicators of lung permeability to water and protein. When lung permeability increases, LPI and W/D ratio increase. In this study, we found that after ischemia-reperfusion, LPI and WID ratio significantly increased and got worse with time, indicating that alveolarcapillary barrier function was impaired after ischemia-reperfusion and deteriorated as reperfusion persisted. Sevoflurane is an inhaled anesthetic commonly used in the clinic, and sevoflurane inhalation itself has no effect on lung tissue but it can alleviate lung injury caused by endotoxin. Suter et al. ${ }^{11}$ found that sevoflurane pretreatment can reduce endotoxin-induced pulmonary edema and inflammatory cell infiltration. In this study, sevoflurane pretreatment reduced the extent of the LPI and W/D increases during reperfusion, suggesting that sevoflurane pretreatment can improve pulmonary vascular permeability after reperfusion and protect lung tissue from ischemia-reperfusion injury.
The alveolar-capillary barrier consists of endothelial cells, pneumocytes and basement membranes between two cells. It is a semipermeable membrane between pulmonary capillaries and the alveolar cavity, limiting the free movement of ions, fluid and a variety of large molecules. There are three main types of cell junctions: tight junctions, adhesions and gap junctions. Tight junctions, also known as occluding junctions, are located at the apical region of the cell membrane and form the closest contact between adjacent cells. They are the 'gateway' that limits solute diffusion through intercellular spaces. ${ }^{4}$

Occludin, a transmembrane protein, and ZO-1, a cytoplasmic protein, are the main components of tight junctions. Occludin is connected to ZO- 1 through the former's C-terminal region, and $\mathrm{ZO}-1$ is then connected to the actin cytoskeleton. Therefore, a signal transduction pathway is established between tight junctions and the cytoskeleton. Stimulating factors can induce cytoskeletal protein activation 
a

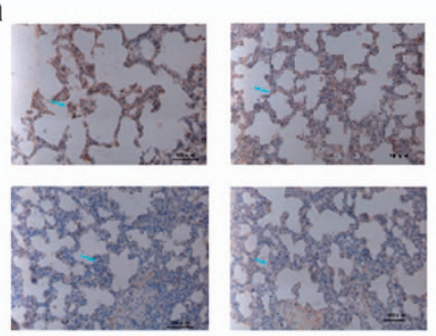

C

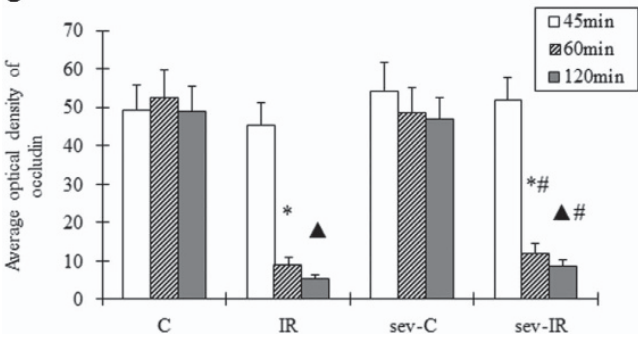

b

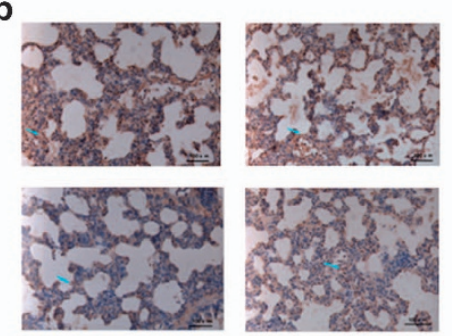

d

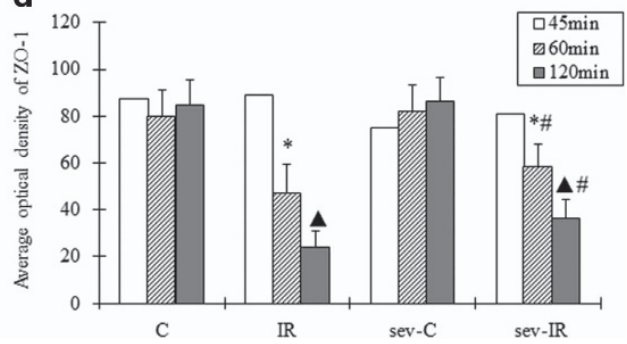

Figure 4 Localization and expression of occludin and ZO-1 in lung tissues following ischemia/reperfusion. The animals were treated as in Figure 1, and the localization and expression of occludin (a) and ZO-1 (b) were determined by immunohistochemistry. (a) and (b) Upper left panel: control; upper right panel: IR for $120 \mathrm{~min}$; lower left panel: sev-C for $120 \mathrm{~min}$; lower right panel: sev-IR for $120 \mathrm{~min}$. (c) Densitometric analysis of occludin in $\mathbf{a}$. (d) ZO-1 expression in $\mathbf{b}$. The data in $\mathbf{c}$ and $\mathbf{d}$ are presented as the mean \pm s.d. of at least three independent experiments ( $n=6$ in each group). ${ }^{*} P<0.05$ vs group $\mathrm{C}$ and group sev-C at 60 -min reperfusion; $\boldsymbol{\Delta} P<0.05$ vs group $\mathrm{C}$ and group sev-C at 120 -min reperfusion; ${ }^{\#} P<0.05$ vs group IR at 60 and 120 -min reperfusion.

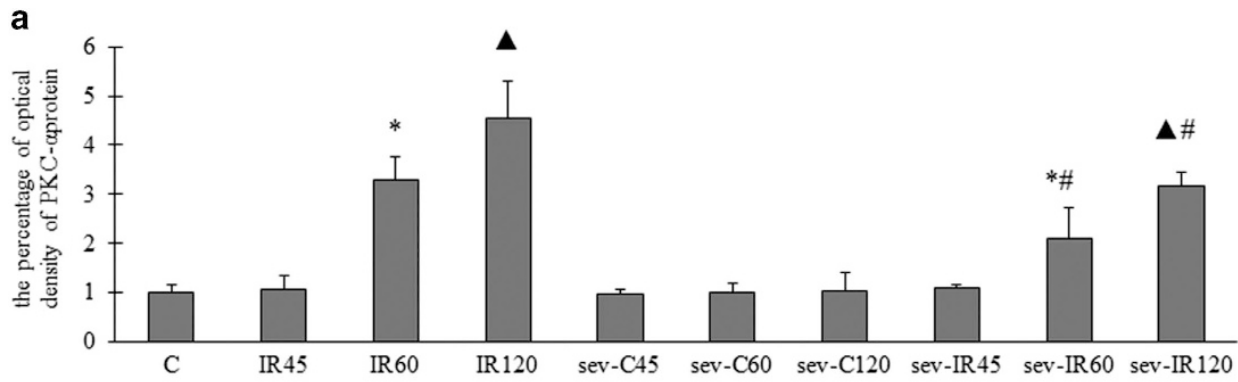

b

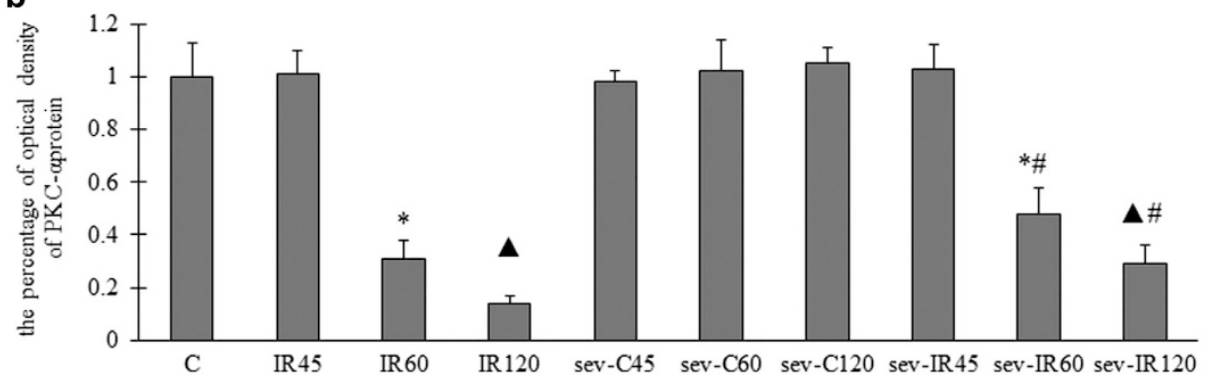

Figure 5 Sevoflurane inhibits PKC- $\alpha$ translocation after reperfusion. The animals were treated as in Figure 1 . Lung tissues were homogenized, and PKC- $\alpha$ was measured by Western blot in the membrane fraction (a) and cytosolic fraction (b). Bands were quantified by densitometry. The data shown are the mean \pm s.d. of at least three independent experiments ( $n=6$ in each group). ${ }^{*} P<0.05$ vs group $C$

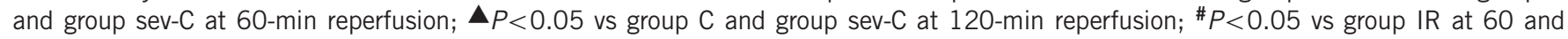
120-min reperfusion.

and then endothelial cell contraction, leading to increased gaps between cells and increased permeability. ${ }^{12}$ The downregulation or redistribution of occludin and $\mathrm{ZO}-1$ increases the permeability between endothelial cells and epithelial cells. ${ }^{13,14}$
Kago et al. ${ }^{13}$ found that in rat brain embolism, occludin and ZO-1 expression in endothelial cells decreased, resulting in increased permeability of the blood-brain barrier. Mark et al. ${ }^{14}$ found that the downregulation or redistribution of occludin 
and ZO-1 in intestinal epithelial cells impaired the function of the intestinal epithelial barrier, causing malabsorption and diarrhea. However, it is not clear whether the regulation of occludin and ZO-1 is related to lung tissue permeability in ischemia-reperfusion injury.

Our data showed that in ischemia-reperfusion injury, lung tissue permeability increased, and occludin and ZO-1 expression decreased, reaching their lowest levels at 120-min reperfusion. These findings indicate that tight junction protein expression is significantly inhibited by ischemia-reperfusion. Transmission electron microscopy revealed disruptions and loosening of tight junctions between the epithelial cells of lung tissues following ischemia/reperfusion, which was most apparent at 120-min post reperfusion. The downregulation of occludin and ZO-1 correlated with the degree of injury to the tight junctions. The changes in occludin and ZO-1 expression in lung tissue correlates to the change in lung tissue permeability, suggesting that structural abnormalities of tight junctions result in alveolar-capillary barrier damage. This is consistent with the findings by Kago et al. ${ }^{13}$ and Mark et al. ${ }^{14}$. Sevoflurane pretreatment increased the expression of occludin and ZO-1 that was depressed by ischemia/reperfusion and improved the integrity of tight junctions and the permeability of the lung tissues under transmission electron microscopy. These findings indicate that sevoflurane may exert the above actions by causing the upregulation of occludin and ZO-1. Sevoflurane can preserve alveolar-capillary barrier function by maintaining integrity of cell junctions. However, it is still not clear why sevoflurane can promote occludin and ZO-1 expression.

To further elucidate the mechanism by which sevoflurane protects lung tissue permeability, we examined the expression of PKC- $\alpha$. PKC- $\alpha$ can trigger cytoskeletal protein contraction in endothelial cells. ${ }^{15,16}$ PKC- $\alpha$ activation has an important role in maintaining endothelial permeability. There are tyrosine and serine/threonine phosphorylation sites at the cytoplasmic end of occludin. Activated PKC- $\alpha$ can bind to those sites and lead to occludin phosphorylation, promoting occludin binding to ZO-1 and then inducing cytoskeletal protein contraction and increasing permeability. PKC- $\alpha$ belongs to the serine/threonine protein kinase family and remains inactive without stimulation. Once activated, PKC- $\alpha$ shifts from the cytoplasm to the cell membrane or cytoskeleton. This translocation is an important indication of PKC- $\alpha$ activation. In different tissues, there are different isoforms of PKC, PKC- $\alpha$ being the major isoform in endothelial cells. ${ }^{17}$ In this study, we found that PKC- $\alpha$ translocation and activation occurred after ischemia-reperfusion injury and correlated to the change in lung tissue permeability. The protective effect of sevoflurane pretreatment on lung tissue permeability was related to PKC- $\alpha$ translocation and activation. Moreover, we found that with increased PKC- $\alpha$ translocation and activation, lung tissue occludin and $\mathrm{ZO}-1$ expression decreased after reperfusion. However, it is not clear whether tight junctions are related to PKC- $\alpha$ translocation and activation.
In this study, we also examined the change in $\mathrm{SpO}_{2}$ during ischemia-reperfusion injury. $\mathrm{SpO}_{2}$ significantly decreased after ischemia-reperfusion, and this change was related to increased lung tissue permeability, which results in pulmonary interstitial edema, exudate accumulation in alveolar space and impaired ventilation/perfusion. $\mathrm{SpO}_{2}$ slightly increased after sevoflurane pretreatment, but the change was not statistically significant Although sevoflurane had a protective effect on lung tissue after reperfusion, it did not improve $\mathrm{SpO}_{2}$. This may have been due to a shorter period of sevoflurane pretreatment or low sevoflurane concentration.

How the expression of occludin and ZO-1 is modulated remains unclear. PKC- $\alpha^{18,19}$ and inflammatory factors ${ }^{20}$ may modulate occludin and ZO-1 expression by acting on the phosphorylation sites of occludin. The current study did not address how sevoflurane modulated occludin and ZO-1 expression via PKC- $\alpha$ and inflammatory factors, which nevertheless remains an important issue and will be addressed in future studies in our laboratory.

In summary, our findings indicate that sevoflurane reduced lung tissue permeability and had a protective effect on lung tissue after ischemia-reperfusion injury. The protective effect of sevoflurane is related to its upregulation of occludin and ZO-1, and inhibition of PKC- $\alpha$ translocation and activation. This study may help in choosing anesthetics and protecting lung function from further damage after ischemia-reperfusion injury.

\section{CONFLICT OF INTEREST}

The authors declare no conflict of interest.

1 Seal JB, Gewertz BL. Vascular dysfunction in ischemia-reperfusion injury. Ann Vasc Surg 2005; 19: 572-584.

2 El Azab SR, Rosseel PM, De Lange JJ, Groeneveld AB, Van Strik R, Van Wijk EM et al. Effect of sevoflurane on the ex vivo secretion of TNF-alpha during and after coronary artery bypass surgery. Eur J Anaesthesiol 2003; 20: 380-384

3 Chai J, Wang DW, Shen J. Effect of sevoflurane on pulmonary oxygen free radicals in ischemia-reperfusion rats. Pract Pharm Clin Rem 2009; 12 : $156-158$.

4 Aijaz S, Balda MS, Matter K. Tight junctions: Molecular architecture and function. Int Rev Cytol 2006; 248: 261-298.

5 Anderson JM, Van Itallie CM. Tight junctions and the molecular basis for regulation of paracellular permeability. Am J Physiol 1995; 269 G467-G475.

6 Dempsey EC, Cool CD, Littler CM. Lung disease and PKCs. Pharmacol Res 2007; 55: 545-559.

7 Shin Y, Yoon SH, Choe EY, Cho SH, Woo CH, Rho JY et al. PMA-induced up-regulation of MMP-9 is regulated by a PKCalpha-NF-kappaB cascade in human lung epithelial cells. Exp Mol Med 2007; 39: 97-105.

8 Vandenbroucke St Amant E, Tauseef M, Vogel SM, Gao XP, Mehta D, Komarova YA et al. PKC $\alpha$ activation of p120-catenin serine 879 phosphoswitch disassembles VE-cadherin junctions and disrupts vascular integrity. Circ Res 2012; 111: 739-749.

9 Hecquet CM, Zhang M, Mittal M, Vogel SM, Di A, Gao X et al. Cooperative interaction of trp melastatin channel TRPM2 with its splice variant TRPM2-S is essential for endothelial cell apoptosis. Circ Res 2014; 114: 469-479.

10 Bellido-Reyes YA, Akamatsu H, Kojima K, Arai H, Tanaka H, Sunamori M. Cytosolic phospholipase A2 inhibition attenuates ischemia-reperfusion injury in an isolated rat lung model. Transplantation 2006; 81: $1700-1707$. 
11 Suter D, Spahn DR, Blumenthal S, Reyes L, Booy C, Z'graggen BR et al. The immunomodulatory effect of sevoflurane in endotoxin-injured alveolar epithelial cells. Anesth Analg 2007; 104: 638-645.

12 Ando-Akatsuka Y, Saitou M, Hirase T, Kishi M, Sakakibara A, Itoh M et al. Interspecies diversity of the occluding sequence: CDNA cloning of human, mouse, dog and rat kangaroo homologues. J Cell Biol 1996; 133: 43-47.

13 Kago T, Takagi N, Date I, Takenaga Y, Takagi K, Takeo S. Cerebral ischemia enhances tyrosine phosphorylation of occludin in brain capillaries. Biochem Biophys Res Commun 2006; 339: 1197-1203.

14 Mark MW, Walsh-Reitz M, Chang EB. Roles of ZO-1, occludin, and actin in oxidant-induced barrier disruption. Am J Physiol Gastrointest Liver Physiol 2006; 290: G222-G231.

15 Sukumaran SK, Prasadarao NV. Escherichia coli K1 invasion increases human brain microvascular endothelial cell monolayer permeability by disassembling vascular-endothelial cadherins at tight junctions. J Infect Dis 2003; 188: 1295-1309.

16 Ferro T, Neumann P, Gertzberg N, Clements R, Johnson A. Protein kinase $\mathrm{Ca}$ mediates endothelial barrier dysfunction induced by TNF-a. Am J Physiol Lung Cell Mol Physiol 2000; 278: L1107-L1117.

17 Huang F, Subbaiah PV, Holian O, Zhang J, Johnson A, Gertzberg N et al. Lysophosphatidylcholine increases endothelial permeability: role of PKCalpha and RhoA cross talk. Am J Physiol Lung Cell Mol Physiol 2005; 289: L176-L185.
18 Clark CA, Thomas LK, Azghani AO. Inhibition of protein kinase $\mathrm{C}$ attenuates Pseudomonas aeruginosa elastase-induced epithelial barrier disruption. Am J Respir Cell Mol Biol 2011; 45: 1263-1271.

19 Andreeva AY, Krause E, Muller EC, Blasig IE, Utepbergenov DI. Protein kinase $C$ regulates the phosphorylation and cellular localization of occludin. J Biol Chem 2001; 276: 38480-38486.

20 Coyne CB, Vanhook MK, Gambling TM, Carson JL, Boucher RC, Johnson LG. Regulation of airway tight junctions by proinflammatory cytokines. $\mathrm{Mol}$ Biol Cell 2002; 13: 3218-3234.

This work is licensed under a Creative Commons Attribution-NonCommercial-NoDerivs 4.0 International License. The images or other third party material in this article are included in the article's Creative Commons license, unless indicated otherwise in the credit line; if the material is not included under the Creative Commons license, users will need to obtain permission from the license holder to reproduce the material. To view a copy of this license, visit http://creativecommons.org/licenses/by-nc-nd/4.0/ 